\title{
Constant Regions in Models of Arithmetic
}

\author{
Tin Lok Wong
}

\begin{abstract}
This paper introduces a new theory of constant regions, which generalizes that of interstices, in nonstandard models of arithmetic. In particular, we show that two homogeneity notions introduced by Richard Kaye and the author, namely, constantness and pregenericity, are equivalent. This led to some new characterizations of generic cuts in terms of existential closedness.
\end{abstract}

\section{Introduction}

Kaye [4] introduced the notions of constant and small intervals for the definition of generic cuts. Kaye and the author [6] simplified this definition to one that omits smallness but uses a two-variable version of constantness. This two-variable version of constantness was called pregenericity in our paper.

Definition Let $Y$ be an indicator on a model $M \models$ PA. A $Y$-interval $\llbracket a, b \rrbracket$ is constant over $c$ if

$$
\forall x \in \llbracket a, b \rrbracket \forall \llbracket u, v \rrbracket \subseteq \llbracket a, b \rrbracket \exists x^{\prime} \in \llbracket u, v \rrbracket(M, x, c) \equiv\left(M, x^{\prime}, c\right) .
$$

A $Y$-interval $\llbracket a, b \rrbracket$ is pregeneric over $c$ if

$$
\forall x, y \in \llbracket a, b \rrbracket \forall \llbracket u, v \rrbracket \subseteq \llbracket a, b \rrbracket \exists x^{\prime}, y^{\prime} \in \llbracket u, v \rrbracket(M, x, y, c) \equiv\left(M, x^{\prime}, y^{\prime}, c\right) .
$$

We show in this paper that constantness and pregenericity actually coincide. In particular, the notion of smallness is redundant in Kaye's original paper [4]. It turns out that looking at intervals with endpoints in the model obscures some features of the notions. So instead, we consider general convex sets with the analogous homogeneity property-we call these constant regions.

After setting up some notation in Section 2, we develop a theory of constant regions in Sections 3-7 parallel to that of interstices. The relationship between the

Received January 2, 2013; accepted June 6, 2013

2010 Mathematics Subject Classification: Primary 03C62; Secondary 03H15

Keywords: constant regions, nonstandard models of arithmetic, interstices, generic cuts, existentially closed models

(C) 2015 by University of Notre Dame 10.1215/00294527-3153615 
two theories is briefly studied in Section 9. In between, it is shown in Section 8 that essentially all cuts inside a constant region are closed under the same definable functions. This reveals a new sense of homogeneity for constant regions. In Section 10, we prove the key theorem of this paper, that all constant intervals are pregeneric.

This supplies useful sufficient conditions for a cut to be generic. In addition to a simplified definition of generic cuts which we present in Section 11, several new characterizations of generic cuts in terms of special cuts and existential closedness are obtained. Specialness is the opposite of genericity that we propose in Section 12. A few elementary facts about existentially closed cuts are included in Section 13. After setting up some lemmas in Section 14 about limits of coded $\omega$-sequences, we prove the remaining characterizations in Section 15.

\section{Preliminaries}

For background information about nonstandard models of arithmetic, we refer the reader to Kaye [3] and Kossak and Schmerl [9]. Our language $\mathscr{L}_{\mathrm{A}}$ contains the symbols $0,1,+, \times,<$. Throughout this paper, we work in a fixed nonstandard model $M \models$ PA. For $c \in M$, let $\operatorname{cl}(c)$ denote the Skolem closure of $c$ in $M$. Unless otherwise stated, "definable" means "definable with parameters." We sometimes write $\mathrm{Q}$ for "there are cofinally many." A cut of $M$ is a nonempty initial segment of $M$ with no maximum element. Informally, we say a convex subset $\Omega \subseteq M$ contains a cut $I$ if inf $\Omega \subseteq I \subseteq \sup \Omega$. For $c \in M$, denote by $\operatorname{Aut}(M, c)$ the stabilizer of $c$ under the automorphism group of $M$.

We assume that the reader is familiar with the general theory of indicators from Kaye [4] and Kaye and Wong [6], [7]. Nevertheless, we repeat a few more important definitions and facts here.

On our model $M$ we have a monotone indicator $Y$ which will be fixed throughout. For each $k \in \mathbb{N}$, set

$$
Y_{k}(x)=(\mu y)(Y(x, y) \geqslant k) .
$$

By definition, the following hold.

(1) $Y$ is a function $M^{2} \rightarrow M$ whose graph is a class. In other words, the set

$$
\{\langle x, y, Y(x, y)\rangle: x, y<b\}
$$

is definable in $M$ for every $b \in M$.

(2) For all $x, y, x^{\prime}, y^{\prime} \in M$, if $(M, x, y) \equiv\left(M, x^{\prime}, y^{\prime}\right)$, then

$$
Y(x, y)>\mathbb{N} \quad \Leftrightarrow \quad Y\left(x^{\prime}, y^{\prime}\right)>\mathbb{N} .
$$

(3) For all $x, y, z \in M$, if $Y(x, y)>\mathbb{N}$, then either $Y(x, z)>\mathbb{N}$ or $Y(z, y)>\mathbb{N}$.

(4) $M \models \forall x, y, u, v(x \leqslant u \wedge v \leqslant y \rightarrow Y(u, v) \leqslant Y(x, y))$.

(5) $M \models \forall x, y(x \geqslant y \rightarrow Y(x, y)=0)$.

Additionally, we require that

(6) $Y_{k}$ is parameter-free definable for each $k \in \mathbb{N}$.

This will be needed in Lemma 5.2 and in Section 13, for example. All naturally occurring indicators $Y$ known to the author satisfy this additional requirement.

Condition (4) above guarantees that the $Y_{k}$ 's are monotone in all arguments. Condition (3) ensures that the family of functions $\left\{Y_{k}: k \in \mathbb{N}\right\}$ is closed under composition, in the sense that

$$
\forall b \in M \forall \ell \in \mathbb{N} \exists k \in \mathbb{N} \forall x<b Y_{\ell}^{2}(x)<Y_{k}(x) .
$$


To see why this holds, note that for all nonstandard $v \in M$ and all $x<b$, we have $Y\left(x, Y_{\ell}^{2}(x)\right) \in \mathbb{N}<Y\left(x, Y_{v}(x)\right)$ by (3), and so $Y_{\ell}^{2}(x)<Y_{v}(x)$. An application of underspill then gives the $k$ we want. In many cases, for example, when $Y$ is definable, one can actually find one $k \in \mathbb{N}$ that works for all $b \in M$.

Recall that double brackets $\llbracket \cdot, \cdot \rrbracket$ are reserved for $Y$-intervals, that is, intervals $[a, b]$ which satisfy $Y(a, b)>\mathbb{N}$. We write $a \ll b$ if $[a, b]$ is a $Y$-interval. A subset of $M$ is $Y$-large if it includes some $Y$-interval. A $Y$-cut is a cut that is closed under $Y_{k}$ for every $k \in \mathbb{N}$. For $d \in M$, define

$$
\begin{aligned}
& M(d)=\{y \in M: Y(d, y) \in \mathbb{N}\} \quad \text { and } \\
& M[d]=\{x \in M: Y(x, d)>\mathbb{N}\} .
\end{aligned}
$$

The monotonicity of $Y$ guarantees that both of these are $Y$-cuts.

It would be helpful, although not necessary, for the reader to be acquainted with the theory of interstices from Bamber and Kotlarski [1].

\section{Constant Regions}

We hope that the word region gives a more positive image than gap and interstice do.

Definition Let $c \in M$. A $Y$-large convex subset $\Omega$ of $M$ is constant over $c$ (with respect to $Y$ ) if

$$
\forall x \in \Omega \forall \llbracket u, v \rrbracket \subseteq \Omega \exists x^{\prime} \in \llbracket u, v \rrbracket(M, x, c) \equiv\left(M, x^{\prime}, c\right) .
$$

A constant region over $c$ is a maximal $Y$-large convex subset of $M$ that is constant over $c$.

Recall that such $x^{\prime}$ can be made to satisfy $u \ll x^{\prime} \ll v$ using the properties of indicators.

With this definition, the model $M$ is required to be recursively saturated by many proofs in this paper. For example, recursive saturation makes sure the union of two intersecting constant convex sets is again constant. Nevertheless, as the reader can verify, most of these still go through if one defines a constant region over $c$ to be a maximal $Y$-large convex set $\Omega$ such that for every $\varphi(x, z) \in \mathscr{L}_{\mathrm{A}}$, there exists $k \in \mathbb{N}$ which satisfies the property

$$
\exists x \in \Omega \varphi(x, c) \Rightarrow \forall[u, v] \subseteq \Omega(Y(u, v)>k \rightarrow \exists x \in[u, v] \varphi(x, c)) .
$$

Over recursive saturation, the two definitions are equivalent (see Lemma 5.2). We avoid this cumbersome definition because we are mainly interested in recursively saturated models of PA.

Kaye [4] showed that constant regions exist in abundance in arithmetically saturated models of PA.

Theorem 3.1 Suppose that $M$ is an arithmetically saturated model of PA, and let $c \in M$. Then every $Y$-interval in $M$ contains a $Y$-large subinterval that is constant over c.

An alternative proof demonstrating the existence of constant regions can be found in an earlier paper by Schmerl [10, Proposition 1.3] using interstices. In fact, we will develop a theory of constant regions mimicking that of interstices. For example, the following lemma has a counterpart for interstices (see Bigorajska, Kotlarski, and Schmerl [2]). The difference is that the lemma, as stated below, no longer needs $\mathbb{N}$ to 
be strong. The use of arithmetic saturation in Theorem 3.1 above is necessary though (see Corollary 8.4).

Lemma 3.2 $\quad$ Assume that the model $M$ is recursively saturated. Let $\Omega$ be a constant region over some element $c \in M$. Then for every $d \in \Omega$,

$$
\inf \Omega<M[d]<M(d)<\sup \Omega .
$$

In particular, both inf $\Omega$ and $\sup \Omega$ are $Y$-cuts.

Proof Pick any $d \in \Omega$. We show that $M[d]>\inf \Omega$. The argument for $\sup \Omega$ is symmetric. Recall that $\Omega$ is $Y$-large. Take $\llbracket r, s \rrbracket \subseteq \Omega$. Using constantness, pick $d^{*} \in \llbracket r, s \rrbracket$ such that $(M, d, c) \equiv\left(M, d^{*}, c\right)$ and $r \ll d^{*} \ll s$. Then find $r^{\prime}, s^{\prime} \in M$ which make $\left(M, d, c, r^{\prime}, s^{\prime}\right) \equiv\left(M, d^{*}, c, r, s\right)$ and thus $r^{\prime} \ll d \ll s^{\prime}$. Such $r^{\prime}, s^{\prime}$ can be found using recursive saturation. Note that $\Omega \cup \llbracket r^{\prime}, s^{\prime} \rrbracket$ has the constantness property over $c$ and that $d \in \Omega \cap \llbracket r^{\prime}, s^{\prime} \rrbracket$. So by the maximality of $\Omega$, we must have $\llbracket r^{\prime}, s^{\prime} \rrbracket \subseteq \Omega$. It follows that $M[d]>r^{\prime}>\inf \Omega$, as required.

This is already sufficient to give us a nice corollary, which in a sense says that we can overspill and underspill across a constant region.

Corollary 3.3 Suppose that $M$ is recursively saturated. Let $\Omega$ be a bounded constant region over a parameter $c \in M$. Then for every $\mathscr{L}_{\mathrm{A}}$-formula $\theta(x, z)$, the following are equivalent.

(a) There are arbitrarily large $x<\inf \Omega$ satisfying $\theta(x, c)$.

(b) There are arbitrarily small $x>\sup \Omega$ satisfying $\theta(x, c)$.

\section{Special Points}

Informally speaking, a special point is a point that can be distinguished from its neighbors by a formula.

Definition Let $c \in M$. An element $w \in M$ is said to be a special point of $M$ over $c$ (with respect to $Y$ ) if there exist $\varphi(x, z) \in \mathscr{L}_{\mathrm{A}}$ and $\llbracket r, s \rrbracket \subseteq M$ such that

- $M \models \varphi(w, c)$;

- $M \models \forall x \in \llbracket r, s \rrbracket \neg \varphi(x, c)$; and

- $w+1=r$ or $s+1=w$.

Lemma 4.1 A constant region contains no special point over the same parameter.

Proof The proof is immediate.

The converse is also true.

Lemma 4.2 Fix a parameter $c \in M$, and suppose that $M$ is recursively saturated. If a convex subset $\Omega \subseteq M$ contains no special point over $c$, then it is constant over $c$.

Proof Suppose that $w \in \Omega$. Let $\llbracket r, s \rrbracket \subseteq \Omega$ in which no $w^{\prime}$ satisfies $(M, w, c) \equiv$ $\left(M, w^{\prime}, c\right)$. Using recursive saturation, find $\varphi(x, z) \in \mathscr{L}_{\mathrm{A}}$ such that

$$
M \models \varphi(w, c) \wedge \forall x \in \llbracket r, s \rrbracket \neg \varphi(x, c) .
$$

Then either $(\max x<r)(\varphi(x, c))$ or $(\min x>s)(\varphi(x, c))$ is a special point over $c$. Such a point is in $\Omega$ because $\Omega$ is convex.

Recall that interstices are convex sets that do not contain definable points. Analogously, constant regions are convex sets that do not contain special points. 


\section{The Type of a Region}

Definition Let $c \in M$, and let $\Omega$ be a constant region over $c$. An $\mathscr{L}_{\mathrm{A}}(c)$-formula $\theta(x, c)$ is said to be satisfied in $\Omega$ if there is an $x \in \Omega$ such that $M \models \theta(x, c)$. Otherwise, it is said to be excluded from $\Omega$. The set of all formulas $\theta(x, c)$ that are satisfied in $\Omega$ is referred to as the type of $\Omega$ over $c$, denoted by $\operatorname{tp}(\Omega / c)$.

It was shown in [6, Section 6] that if $Y(x, y)=z$ is a single formula, then there are countably many types of constant regions over any given $c$.

Lemma 5.1 If $M$ is recursively saturated, then $\operatorname{tp}(\Omega / c)$ is coded for all $c \in M$ and all constant regions $\Omega$ over $c$.

Proof For any $\llbracket r, s \rrbracket \subseteq \Omega$, we have

$$
\operatorname{tp}(\Omega / c)=\{\theta(x, c): M \models \exists x \in \llbracket r, s \rrbracket \theta(x, c)\} .
$$

Clearly, a formula satisfied in a constant region is densely satisfied with respect to the indicator. Using recursive saturation, we can get a uniform bound on this density.

Lemma 5.2 Suppose that $M$ is recursively saturated, and let $c \in M$. If $\theta(x, c)$ is an $\mathscr{L}_{\mathrm{A}}(c)$-formula satisfied in a constant region $\Omega$ over $c$, then there is $k \in \mathbb{N}$ such that

$$
\forall[u, v] \subseteq \Omega(Y(u, v)>k \rightarrow \exists x \in[u, v] \theta(x, c)) .
$$

Proof Pick any $\llbracket r, s \rrbracket \subseteq \Omega$. We know that $\theta(x, c)$ is satisfied in $\llbracket r, s \rrbracket$ by constantness. Hence recursive saturation gives $k \in \mathbb{N}$ such that

$$
\forall u \in \llbracket r, s \rrbracket \exists x \in\left[u, Y_{k}(u)\right] \theta(x, c) .
$$

This $k$ is what we want because both

$$
\begin{aligned}
& (\max u<r)\left(\forall x \in\left[u, Y_{k}(u)\right] \neg \theta(x, c)\right) \quad \text { and } \\
& (\min u>s)\left(\forall x \in\left[u, Y_{k}(u)\right] \neg \theta(x, c)\right)
\end{aligned}
$$

are special points over $c$, and thus have to lie outside $\Omega$, if they exist.

In countable recursively saturated models $M \models \mathrm{PA}$, the type of a constant region determines its orbit under the action of the automorphism group of $M$.

Proposition 5.3 Suppose that $M$ is a countable recursively saturated model of PA. Let $c \in M$. If $\Omega_{1}$ and $\Omega_{2}$ are constant regions with the same type over $c$, then there exists $g \in \operatorname{Aut}(M, c)$ such that $g\left(\Omega_{1}\right)=\Omega_{2}$.

Proof If $x \in \Omega_{1}$ and $\llbracket r, s \rrbracket \subseteq \Omega_{2}$, then recursive saturation says that there is $x^{\prime} \in \llbracket r, s \rrbracket$ which has the same type as $x$ over $c$.

\section{Maxima and Minima}

By underspill, if a formula $\theta(x, c)$ excluded from a constant region $\Omega$ is satisfied below $\Omega$, then $(\max x<\Omega)(\theta(x, c))$ exists. Similarly, by overspill, if $\theta(x, c)$ is excluded from $\Omega$ but is satisfied above $\Omega$, then $(\min x>\Omega)(\theta(x, c))$ exists. These maxima and minima are clearly special points over $c$. 
Lemma 6.1 Suppose that $M$ is recursively saturated. Let $c \in M$, and let $\Omega$ be a constant region over $c$. Then

$$
\inf \Omega=\sup \{(\max x<\Omega)(\theta(x, c)): \theta \text { is satisfied below but not in } \Omega\} .
$$

If, in addition, the region $\Omega$ is bounded, then we also have

$$
\sup \Omega=\inf \{(\min x>\Omega)(\theta(x, c)): \theta \text { is satisfied above but not in } \Omega\} .
$$

Proof We concentrate on $\inf \Omega$ - the $\operatorname{proof}$ for $\sup \Omega$ is symmetric. Pick $w \in \inf \Omega$. By Lemma 4.2, we can assume that $w$ is a special point over $c$ without loss of generality. Let $\theta(x, c)$ witness the specialty of $w$. There are two possibilities.

If $\theta(x, c)$ is excluded from $\Omega$, then

$$
(\max x<\Omega)(\theta(x, c)) \geqslant w .
$$

So we are done in this case.

Suppose that $\theta(x, c)$ is satisfied in $\Omega$. Using Lemma 5.2, find $k \in \mathbb{N}$ such that

$$
\forall[u, v] \subseteq \Omega(Y(u, v)>k \rightarrow \exists x \in[u, v] \theta(x, c)) .
$$

Depending on the behavior of $\theta(x, c)$ around $w$, we have

$$
\begin{aligned}
& \text { either }(\max u<\Omega)(\exists v(Y(u, v)>k \wedge \forall x \in[u, v] \neg \theta(x, c))) \geqslant w \\
& \quad \text { or }(\max v<\Omega)(\exists u(Y(u, v)>k \wedge \forall x \in[u, v] \neg \theta(x, c)))+1 \geqslant w .
\end{aligned}
$$

This lemma implies that there are coded monotone sequences of special points that converge to a bounded constant region from either side. An interstitial counterpart of this can be found in [1].

Corollary 6.2 Assume that $M$ is recursively saturated. Let $c \in M$, and let $\Omega$ be a constant region over $c$. Then there is $\alpha \in M$ such that

- $(\alpha)_{n}$ is a special point over $c$ for every $n \in \mathbb{N}$;

- $(\alpha)_{n} \leqslant(\alpha)_{n+1}$ for every $n \in \mathbb{N}$; and

- $\sup \left\{(\alpha)_{n}: n \in \mathbb{N}\right\}=\inf \Omega$.

If, in addition, the region $\Omega$ is bounded, then there is $\beta \in M$ such that

- $(\beta)_{n}$ is a special point over $c$ for every $n \in \mathbb{N}$;

- $(\beta)_{n} \geqslant(\beta)_{n+1}$ for every $n \in \mathbb{N} ;$ and

- $\inf \left\{(\beta)_{n}: n \in \mathbb{N}\right\}=\sup \Omega$.

Proof Fix a recursive enumeration $\left(\theta_{n}(x, z)\right)_{n \in \mathbb{N}}$ of $\mathscr{L}_{\mathrm{A}}$-formulas, and pick any $\llbracket r, s \rrbracket \subseteq \Omega$. Using recursive saturation, find $\alpha \in M$ such that for all $n \in \mathbb{N}$,

$$
(\alpha)_{n}= \begin{cases}(\max x<r)\left(\theta_{n}(x, c)\right), & \text { if } \theta_{n}(x, c) \text { is satisfied below } r \text { but not in } \llbracket r, s \rrbracket ; \\ 0, & \text { otherwise. }\end{cases}
$$

If $\Omega$ is bounded above, say, by the special point $b$, then we may take $\beta$ to be an element of $M$ which satisfies

$$
(\beta)_{n}= \begin{cases}(\min x>s)\left(\theta_{n}(x, c)\right), & \text { if } \theta_{n}(x, c) \text { is satisfied above } s \text { but not in } \llbracket r, s \rrbracket ; \\ b, & \text { otherwise }\end{cases}
$$

for all $n \in \mathbb{N}$. Such $\beta$ exists by recursive saturation. 


\section{Infimum and Supremum}

Given a cut $I$, it is useful to know how the definable functions under which $I$ is closed relate to points of the forms

$$
(\max x \in I)(\theta(x, c)) \quad \text { and } \quad(\min x>I)(\theta(x, c)),
$$

where $\theta(x, z) \in \mathscr{L}_{\mathrm{A}}$. To turn a definable function into a formula, one iterates the function. This is what we do in the following proof. More applications of this trick can be found in the proofs of Theorem 8.1 and Proposition 9.5.

Lemma 7.1 Let I be a cut. Denote by $\mathfrak{G}_{I}$ the set of all increasing functions $F: M \rightarrow M$ such that $F$ is definable over $c$ and $I$ is not closed under $F$. Suppose that $\mathfrak{E}_{I}$ is nonempty.

(a) For every $F \in \mathfrak{E}_{I}$, there is a formula $\theta(x, z) \in \mathscr{L}_{\mathrm{A}}$ such that

$$
F((\max x \in I)(\theta(x, c)))=(\min x>I)(\theta(x, c)) .
$$

(b) If $u=(\max x \in I)(\theta(x, c))$ for some $\theta(x, z) \in \mathscr{L}_{\mathrm{A}}$, then there is $F \in \mathfrak{S}_{I}$ such that $F(u)=u$.

(c) If $v=(\min x>I)(\theta(x, c))$ for some $\theta(x, z) \in \mathscr{L}_{\mathrm{A}}$, then there is $F \in \mathfrak{S}_{I}$ such that $F(I) \leqslant v$.

Proof First consider (a). Define $G(0)=0$ and

$$
G(u+1)= \begin{cases}F(G(u)), & \text { if } F(G(u))>G(u) ; \\ G(u)+1, & \text { otherwise. }\end{cases}
$$

If $\operatorname{Im}(G) \cap I \subseteq_{\mathrm{cf}} I$, then $I$ is closed under $F$, which is not the case. So both $(\max v \in I)(v \in \operatorname{Im}(G))$ and $(\min v>I)(v \in \operatorname{Im}(G))$ exist, and

$$
F((\max v \in I)(v \in \operatorname{Im}(G)))=(\min v>I)(v \in \operatorname{Im}(G)),
$$

as required.

For (b) and (c), use the function $F$ defined by

$$
F(x)= \begin{cases}\left(\mu x^{\prime} \geqslant x\right)\left(\theta\left(x^{\prime}, c\right)\right), & \text { if } \exists x^{\prime} \geqslant x \theta\left(x^{\prime}, c\right) ; \\ G(x), & \text { otherwise, }\end{cases}
$$

where $G$ is some fixed element of $\mathfrak{E}_{I}$.

The reader is invited to unravel the following corollary to see what picture the lemma above gives.

Corollary 7.2 Let I be a cut. Denote by $\overline{\mathfrak{E}}_{I}$ the set of all definable functions over $c$ under which I is not closed. If $\overline{\mathfrak{F}}_{I}$ is nonempty, then

$$
\begin{aligned}
& \bigcup\left\{\sup \left(F^{-1}(I) \cap I\right): F \in \overline{\mathfrak{E}}_{I}\right\} \\
& \quad=\sup \left\{(\max x \in I)(\theta(x, c)): \theta \in \mathscr{L}_{\mathrm{A}} \text { for which the max exists }\right\}
\end{aligned}
$$

and

$$
\begin{aligned}
& \bigcap\left\{\sup F(I): F \in \overline{\mathfrak{E}}_{I}\right\} \\
& =\inf \left\{(\min x>I)(\theta(x, c)): \theta \in \mathscr{L}_{\mathrm{A}} \text { for which the min exists }\right\} .
\end{aligned}
$$

Lemma 7.1(a) tells us that we can transfer closure conditions between the infimum and the supremum of a constant region. This is similar to the situation for interstices. 
Proposition 7.3 Suppose that $M$ is a recursively saturated model of PA. Let $\Omega$ be a constant region over a parameter $c \in M$. Then the following are equivalent for a function $F: M \rightarrow M$ definable over $c$.

(a) The cut inf $\Omega$ is not closed under $F$.

(b) The cut $\sup \Omega$ is not closed under $F$.

(c) There is a special point $w$ over $c$ in $\inf \Omega$ such that $F(w)>\sup \Omega$.

Proof Without loss of generality, suppose that $F$ is nondecreasing. It is clear that (c) $\Rightarrow$ (a) and (c) $\Rightarrow$ (b). To prove the converses, note that if $I$ is a $Y$-cut, then all points of the forms

$$
(\max x \in I)(\theta(x, c)) \quad \text { and } \quad(\min x>I)(\theta(x, c)),
$$

where $\theta(x, z)$ is an $\mathscr{L}_{\mathrm{A}}$-formula, are special over $c$. Therefore, we are done by Lemmas 4.1 and 7.1(a).

Corollary 7.4 Assume that $M$ is recursively saturated. Let $c \in M$, and let $\Omega$ be a constant region over $c$. Then inf $\Omega$ and $\sup \Omega$ are closed under the same $\mathscr{L}_{\mathrm{A}}$-definable functions over $c$.

Consequently, if $\Omega$ is unbounded, then inf $\Omega \prec \sup \Omega$. The converse, however, is not true. The most obvious example is when $Y$ is the indicator for elementary cuts, in which case Lemma 3.2 makes both inf $\Omega$ and sup $\Omega$ elementary. Section 6 of Bigorajska, Kotlarski, and Schmerl [2] contains examples where sup $\Omega$ is not elementary in $M$. (See Section 9 for some explanations of why that paper is relevant.)

\section{Closedness under Functions}

We investigate, in this section, under which functions the cuts inf $\Omega$ and $\sup \Omega$ are closed for a constant region $\Omega$. It turns out that our result works for all the $Y$-cuts in between too.

Theorem 8.1 Suppose that $M$ is a recursively saturated model of PA. Let $\Omega$ be a constant region over some element $c \in M$. Then for all functions $F: M \rightarrow M$ definable over $c$, the following are equivalent.

(a) There exists a $Y$-cut I in $\Omega$ that is closed under $F$.

(b) All $Y$-cuts $I$ in $\Omega$ are closed under $F$.

(c) There exists $k \in \mathbb{N}$ such that for every $x \in \Omega$, we have $F(x) \leqslant Y_{k}(x)$.

Proof Clearly (c) $\Rightarrow$ (b) and (b) $\Rightarrow$ (a). So it remains to prove (a) $\Rightarrow$ (c).

Let $I$ be a $Y$-cut in $\Omega$ that is closed under $F$. By Proposition 7.3, if $I=\sup \Omega$, then $\inf \Omega$ is closed under $F$ too. So it is safe to assume that $I \neq \sup \Omega$, and hence that $I$ is a proper cut of $M$. We may also assume that $x<F(x) \leqslant F(x+1)$ for all $x \in M$.

Define $G(u)=F^{u}(0)$. Since $I$ is closed under $F$, we know that $\operatorname{Im}(G) \cap I \subseteq_{\mathrm{cf}} I$. If $I=\inf \Omega$, then this overspills. Otherwise, one directly sees that $\operatorname{Im}(G) \cap \Omega \neq \varnothing$. Using Lemma 5.2, find $\ell \in \mathbb{N}$ such that

$$
\operatorname{Im}(G) \cap\left[d+1, Y_{\ell}(d)\right] \neq \varnothing
$$

for all $d \in \Omega$. We claim that $F(x) \leqslant Y_{\ell}^{2}(x)$ for every $x \in \Omega$. Since $I \neq M$ and the $Y_{k}$ 's are closed under composition, this will imply the existence of $k \in \mathbb{N}$ that makes 
$F(x) \leqslant Y_{k}(x)$ for all small enough $x \in \Omega$. An application of constantness together with Lemma 3.2 will then finish the proof.

Pick any $x \in \Omega$. Let $d=(\max w \in \operatorname{Im} G)(w \leqslant x)$. Then

$$
\begin{aligned}
F(x) & \leqslant F(F(d)) \quad \text { since } x<F(d) \text { by maximality, } \\
& \leqslant Y_{\ell}(F(d)) \quad \text { since } F(d) \in \operatorname{Im} G, \\
& \leqslant Y_{\ell}^{2}(d) \quad \text { since } d \in \operatorname{Im} G \text { and so } F(d) \leqslant Y_{\ell}(d), \\
& \leqslant Y_{\ell}^{2}(x) \quad \text { since } d \leqslant x,
\end{aligned}
$$

as required.

This theorem can be restated in terms of domination of functions.

Definition Let $I \subseteq_{\mathrm{e}} M$ and $F, G: M \rightarrow M$. We say that $F$ dominates $G$ on $I$ if there is an element of $I$ above which all $x \in I$ satisfy $F(x) \geqslant G(x)$.

Corollary 8.2 Assume that our model $M$ is recursively saturated. Fix a parameter $c \in M$ and a constant region $\Omega$ over $c$. Let $I$ be $a Y$-cut in $\Omega$ and $F: M \rightarrow M$ that is $\mathscr{L}_{\mathrm{A}}$-definable over $c$. Then $I$ is closed under $F$ if and only if $F$ is dominated by $Y_{k}$ on I for some $k \in \mathbb{N}$.

It is important that the function $F$ above is definable over the parameter $c$ alone. For instance, with extra parameters, we can find faster growing functions under which inf $\Omega$ is closed (see the proof of Lemma 14.2 and Corollary 6.2). Actually, the proof of Lemma 14.2 shows that inf $\Omega \neq M(d)$ for any $d \in \operatorname{cl}(c)$ over which $Y$ is definable. However, it is not hard to find an indicator $Y$ for which $M(0)$ is the infimum of a constant region, especially when $M(0)=\mathbb{N}$.

Theorem 8.1 puts a tight restriction on unbounded constant intervals. For example, it tells us that if a constant region $\Omega$ is unbounded, then all $Y$-cuts in $\Omega$ must be elementary. So not many constant regions can be unbounded. The next corollary further supports this claim. Some resemblance with the proof of Proposition 6.5 in our previous paper [6] may be observed.

Corollary 8.3 Suppose that $M$ is recursively saturated and that the indicator $Y$ is definable over a parameter $c \in M$. If $\Omega$ is a constant region over the same parameter $c$, then $\Omega$ must be bounded.

Proof Suppose not. Then by Corollary 8.2, every definable function over $c$ is dominated by $Y_{k}$ on $M$ for some $k \in \mathbb{N}$. This is impossible. Consider, for example, the function $F: x \mapsto Y_{x}(x)+1$. It is definable over $c$, and for every $x>k$, we have

$$
F(x)=Y_{x}(x)+1>Y_{x}(x) \geqslant Y_{k}(x) .
$$

Thus, no $Y_{k}$ can dominate $F$ on $M$.

Another piece of information that Theorem 8.1 provides is the necessity of arithmetic saturation in Theorem 3.1. This is similar to [6, Proposition 5.13].

Corollary 8.4 Assume recursive saturation of our model $M$. Suppose that there is a $Y$-interval $\llbracket a, b \rrbracket$ in which we can find for each $c \in M$ a subinterval that is constant over c. Then $\mathbb{N}$ is strong in $M$. 
Proof Fix a code $Y$ for the graph of the indicator below $b$. Let $c \in M$ be a code for a function $f: \mathbb{N} \rightarrow M$. Using the hypothesis, find $\llbracket r, s \rrbracket \subseteq \llbracket a, b \rrbracket$ that is constant over $\langle c, Y\rangle$. Overspill then gives a nonstandard $v \in M$ which satisfies

$$
M \models \forall x<b \exists y Y(x, y)>v .
$$

We claim that for all $m \in \mathbb{N}$,

$$
f(m)>\mathbb{N} \quad \Leftrightarrow \quad f(m)>\min (Y(r, s), v) .
$$

Let $m \in \mathbb{N}$ for which $f(m)>\mathbb{N}$. If $f(m)>v$, then we are done. So suppose that $f(m) \leqslant v$. Consider the function $F$ defined by

$$
F(x)=(\max y)(Y(x, y)<f(m)) .
$$

By the choice of $v$, we know that $F(x)$ exists for every $x<b$. Note that $F$ is monotone wherever it is defined.

Suppose that we have $k \in \mathbb{N}$ and $x<b$ such that $F(x) \leqslant Y_{k}(x)$. Then

$$
F(x) \leqslant Y_{k}(x)=(\mu y)(Y(x, y) \geqslant k) \in M(x),
$$

and so $F(x)+1 \in M(x)$ too. However, by the maximality of $F(x)$, we must have $Y(x, F(x)+1) \geqslant f(m)>\mathbb{N}$. This contradicts the definition of $M(x)$. Therefore $F(x)>Y_{k}(x)$ for all $k \in \mathbb{N}$ and all $x<b$.

Let $\Omega$ be the constant region over $\langle c, Y\rangle$ around $\llbracket r, s \rrbracket$. Corollary 8.2 says that $\inf \Omega$ is not closed under $F$. Using Proposition 7.3, find $w \in \inf \Omega$ such that $F(w)>\sup \Omega$. Note that $[r, s] \subseteq \Omega \subseteq[w, F(w)]$ as a result. Therefore,

$$
Y(r, s) \leqslant Y(w, F(w))<f(m),
$$

as required.

The role of the strength of $\mathbb{N}$ in the context of interstices was studied in Bamber and Kotlarski [1] and Bigorajska, Kotlarski, and Schmerl [2].

\section{Interstices}

The word interstice was introduced by Bamber and Kotlarski in [1], although the notion already appeared in Kaye, Kossak, and Kotlarski [5].

Definition An interstice over $c$ is a maximal nonempty convex subset of $M$ that contains no point from $\operatorname{cl}(c)$. Denote by $\mathfrak{F}_{\Lambda}$ the set of all functions definable over $c$ under which inf $\Lambda$ is closed. The interstitial gap around an element $d \in \Lambda$ (with respect to $\Lambda$ ) is defined to be

$$
\left\{x \in M: F(x)>d \text { and } G(d)>x \text { for some } F, G \in \mathfrak{F}_{\Lambda}\right\} .
$$

Each interstice can, in a sense, be considered as a constant region for some local indicator. This is summarized in the next two propositions. The first of these was proved in [5], while the second appeared in [10].

Proposition 9.1 Let $M$ be an arithmetically saturated model of PA. Then for all $c \in M$ and all interstices $\Lambda$ over $c$, the set $\mathfrak{F}_{\Lambda}$ is coded in $M$ as a set of Gödel numbers.

Proposition 9.2 Suppose that $M$ is a recursively saturated model of PA. Let $\Lambda$ be an interstice over an element $c \in M$. If $x \in \Lambda$ and $[u, v] \subseteq \Lambda$ such that $F(u)<v$ for all $F \in \mathfrak{F}_{\Lambda}$, then there exists $x^{\prime} \in[u, v]$ making $(M, x, c) \equiv\left(M, x^{\prime}, c\right)$. 
More explicitly, if the model $M$ is arithmetically saturated, then given an interstice $\Lambda$ over a parameter $c \in M$, we can make $\Lambda$ into a constant region over $c$ by setting $Y_{k}$ to be the $k$ th element of $\mathfrak{F}_{\Lambda}$ in the coded sequence that Proposition 9.1 provides. Note, however, that our definability assumption on the indicator mostly fails for $Y_{k}$ 's obtained in this way.

Clearly, constant regions have to be subsets of interstices. If a constant region $\Omega$ is included in an interstice $\Lambda$, then $Y_{k} \in \mathfrak{F} \Lambda$ for every $k \in \mathbb{N}$. So by Theorem 3.1, every arithmetically saturated model of PA contains an interstice $\Lambda$ for which $Y_{k} \in \mathfrak{F} \Lambda$ for all $k \in \mathbb{N}$. Conversely, if an interstice $\Lambda$ makes $Y_{k} \in \mathfrak{F}_{\Lambda}$ for every $k \in \mathbb{N}$, then, assuming the model $M$ is arithmetically saturated, it includes a constant region for $Y$ by Theorem 3.1. Furthermore, we know exactly when the inclusion between a constant region and an interstice is proper.

Proposition 9.3 Suppose that $M$ is recursively saturated. Let $\Omega$ be a constant region over an element $c \in M$, and let $\Lambda$ be the interstice over c containing $\Omega$. The following are equivalent:

(a) $\Omega=\Lambda$;

(b) $\inf \Omega=\inf \Lambda$;

(c) inf $\Omega$ is closed under all functions in $\mathfrak{F}_{\Lambda}$;

(d) $\forall F \in \mathfrak{F}_{\Lambda} \exists k \in \mathbb{N} \forall x \in \Lambda Y_{k}(x) \geqslant F(x)$.

Proof The implication (a) $\Rightarrow$ (d) is part of Theorem 8.1 , and (d) $\Rightarrow$ (c) is an easy exercise. We will prove (c) $\Rightarrow$ (b) and (b) $\Rightarrow$ (a).

Suppose that (b) is false. Using Lemma 6.1, find an $\mathscr{L}_{\mathrm{A}}$-formula $\theta(x, z)$ such that $\theta(x, c)$ is satisfied below but not in $\Omega$, and

$$
(\max x<\Omega)(\theta(x, c)) \in \Lambda \cap \inf \Omega .
$$

Call this maximum $w$. Define $F(x)=\left(\mu x^{\prime}>x\right)\left(\theta\left(x^{\prime}, c\right)\right)$. Then $F \in \mathfrak{F} \Lambda$ and $F(w)>\Omega$. So (c) is false.

Now, suppose that (b) holds. We show that $\Lambda$ has the constantness property over $c$, and so (a) holds because of the maximality of $\Omega$. Pick $x \in \Lambda$ and $\llbracket u, v \rrbracket \subseteq \Lambda$. We claim that $F(u)<v$ for every $F \in \mathfrak{F}_{\Lambda}$. Fix $F \in \mathfrak{F}_{\Lambda}$. Let $k \in \mathbb{N}$ such that $Y_{k}$ dominates $F$ on inf $\Omega$. Note that there is a point in $\operatorname{cl}(c) \cap \inf \Omega$ above which all $x \in \inf \Omega$ satisfy $Y_{k}(x) \geqslant F(x)$. Thus actually, we have $Y_{k}(x) \geqslant F(x)$ for all $x \in \Lambda$, because $\Lambda$ does not contain any definable point over $c$. In particular, $F(u) \leqslant Y_{k}(u)<v$. Since the choice of $F \in \mathfrak{F}_{\Lambda}$ is arbitrary, Proposition 9.2 tells us there exists $x^{\prime} \in \llbracket u, v \rrbracket$ such that $(M, x, c) \equiv\left(M, x^{\prime}, c\right)$, as required.

Consequently, provided a constant region is not an interstice, it is always a proper subset of an interstitial gap.

Corollary 9.4 Fix $c \in M$, and suppose that $M$ is recursively saturated. Let $\Omega$ be a constant region over $c$ that is strictly included in an interstice $\Lambda$ over $c$. Then there is an interstitial gap $\lambda \subseteq \Lambda$ which satisfies

$$
\inf \lambda<\inf \Omega<\sup \Omega<\sup \lambda \text {. }
$$

Proof Combine Propositions 9.3(c) and 7.3.

It is clear that constant regions in different interstices cannot have the same type. The next proposition says that if the model is recursively saturated, then distinct constant 
regions of the same type must live in distinct interstitial gaps. The proof we give resembles that of Ehrenfeucht's lemma (see [9, Theorem 1.7.2]).

Proposition 9.5 Assume that $M$ is recursively saturated. Fix an element $c \in M$ and an interstice $\Lambda$ over $c$. Let $\Omega$ and $\Omega^{\prime}$ be constant regions over $c$ inside $\Lambda$ such that $\operatorname{tp}(\Omega / c)=\operatorname{tp}\left(\Omega^{\prime} / c\right)$ and $\Omega<\Omega^{\prime}$. Then $F(\Omega)<\Omega^{\prime}$ for all $F \in \mathfrak{F} \Lambda$.

Proof Let $d \in \Omega$ and $d^{\prime} \in \Omega^{\prime}$. Suppose that we can find an increasing $F \in \mathfrak{F} \Lambda$ such that $F(d) \geqslant d^{\prime}$. Define $G(u)=F^{u}(0)$. Since $F \in \mathfrak{F}_{\Lambda}$, we know that $\operatorname{Im}(G) \cap \inf \Lambda \subseteq_{\mathrm{cf}} \inf \Lambda$. So by overspill, there are images of $G$ inside $\Lambda$. However, inf $\Omega$ cannot be closed under $F$ by Corollary 7.4. Thus $\operatorname{Im}(G) \cap \inf \Omega \nsubseteq_{\text {cf }} \inf \Omega$. Let $u \in M$ be maximum such that $G(u)<\Omega$. Note that $\operatorname{Im}(G) \cap \Omega=\varnothing$ by overspill. So $G(u+1)>\sup \Omega>d$. This implies that

$$
G(u+2)=F(G(u+1)) \geqslant F(d) \geqslant d^{\prime} .
$$

Since $\operatorname{tp}(\Omega / c)=\operatorname{tp}\left(\Omega^{\prime} / c\right)$, we know that $\operatorname{Im}(G) \cap \Omega^{\prime}=\varnothing$. Hence $G(u+2)>\Omega^{\prime}$. Putting everything together, we obtain

$$
\begin{aligned}
& u+1=(\min x)(G(x)>\Omega) \quad \text { and } \\
& u+2=(\min x)\left(G(x)>\Omega^{\prime}\right) .
\end{aligned}
$$

This contradicts the hypothesis that $\operatorname{tp}(\Omega / c)=\operatorname{tp}\left(\Omega^{\prime} / c\right)$.

\section{Pregeneric Intervals}

We are ready for the key theorem of this paper, that all constant intervals are pregeneric over the same parameter.

Theorem 10.1 Suppose that $M$ is a countable recursively saturated model of PA. Fix $c \in M$ and a constant region $\Omega$ over $c$. If $x, y \in \Omega$ and $\llbracket r, s \rrbracket \subseteq \Omega$, then there are $x^{\prime}, y^{\prime} \in \llbracket r, s \rrbracket$ such that $(M, x, y, c) \equiv\left(M, x^{\prime}, y^{\prime}, c\right)$.

Proof By recursive saturation, it suffices to show that the type

$$
p(u, v)=\left\{\varphi(u, v, c) \leftrightarrow \varphi(x, y, c): \varphi \in \mathscr{L}_{\mathrm{A}}\right\}+\{u, v \in[r, s]\}
$$

is finitely satisfied in $M$. Pick $\varphi(u, v, w) \in \mathscr{L}_{\mathrm{A}}$, and suppose that $M \models \varphi(x, y, c)$. Define

$$
F(u)=(\mu v>u)\left(\exists u^{\prime}, v^{\prime} \in[u, v] \varphi\left(u^{\prime}, v^{\prime}, c\right)\right) .
$$

Note that $F$ is monotone. If $\inf \Omega$ is not closed under $F$, then by Proposition 7.3, there is $w \in \inf \Omega$ such that $F(w)>\sup \Omega$. This contradicts the existence of $x$ and $y$. So inf $\Omega$ must be closed under $F$. Theorem 8.1 then implies that $F(r) \leqslant s$. Therefore, $M \models \exists u, v \in \llbracket r, s \rrbracket \varphi(u, v, c)$, as required.

The issues for small intervals are more delicate. It is known that small intervals are not closed under taking subintervals (see Wong [11]). Therefore, not all pregeneric intervals are small. Since the proof is short and has not appeared elsewhere in the literature, we include it here.

Definition An interval $\llbracket a, b \rrbracket$ is small over $c$ if $Y(a, b)<x$ for each nonstandard $x \in \operatorname{cl}(a, c)$.

Proposition 10.2 (Kaye) Let $c \in M$. Then every $Y$-interval $\llbracket a, b \rrbracket$ contains $a$ $Y$-large subinterval that is not small over $c$. 
Proof Without loss of generality, assume that $a$ is nonstandard. Let $d \in \llbracket a, b \rrbracket$ which satisfies $a \ll d \ll b$. Choose a nonstandard $m \in M$ such that

$$
m<\min \left\{\left\lfloor\log _{2}(a)\right\rfloor,\left\lfloor\log _{2}(d-a)\right\rfloor, Y(d, b)\right\} .
$$

Note that $2^{m}<2^{\left\lfloor\log _{2}(a)\right\rfloor} \leqslant a$. So there is an odd $w \in M$ such that $2^{m} w<a$. Let

$$
x=(\max w)\left(\exists y(w=2 y+1) \wedge 2^{m} w<a\right) .
$$

Then $2^{m}(x+2) \geqslant a$ by the maximality of $x$. Also,

$$
2^{m}(x+2)=2^{m} x+2^{m+1}<a+2^{\left\lfloor\log _{2}(d-a)\right\rfloor} \leqslant a+d-a=d .
$$

Hence $2^{m}(x+2) \in \llbracket a, d \rrbracket$. Let $a^{\prime}=2^{m}(x+2)$. Since $a^{\prime} \leqslant d \ll b$, we know that $\llbracket a^{\prime}, b \rrbracket$ is a $Y$-interval. However, by the monotonicity of $Y$,

$$
Y\left(a^{\prime}, b\right) \geqslant Y(d, b)>m=(\max u)\left(2^{u} \mid a^{\prime}\right) \in \operatorname{cl}\left(a^{\prime}, c\right) \backslash \mathbb{N} .
$$

Therefore $\llbracket a^{\prime}, b \rrbracket$ is not small over $c$.

\section{Generic Cuts}

We can thus redefine generic cuts as follows.

Definition A $Y$-cut $I$ is generic if for every $c \in M$, there exists a constant region over $c$ that contains $I$.

Corollary 11.1 Suppose that $M$ is recursively saturated, and let I be a $Y$-cut. Then $I$ is generic if and only if over no parameter in $M$ is I a limit of special points.

Kaye [4] showed that if $M$ is a countable arithmetically saturated model of PA, then the class of generic cuts is the smallest comeager set in the space of $Y$-cuts that is invariant under the action of $\operatorname{Aut}(M)$. This is a simple consequence of Theorem 3.1. In particular, the generic cuts form a dense subset of the space under such hypotheses.

Recall that two cuts $I, J$ are said to be conjugate over an element $c \in M$ exactly when $(M, I, c) \cong(M, J, c)$. On the one hand, Kaye and Wong [6] showed using a back-and-forth argument that—provided $M$ is countable—generic cuts in the same constant region are all conjugate. On the other hand, a constant region is the maximal convex subset of $M$ in which all generic cuts are conjugate. Compare the next proposition with the results in [6, Section 6].

Proposition 11.2 Assume that $M$ is recursively saturated. Fix $c \in M$. Let I be a generic cut, and let $\Omega$ be the constant region over c containing I. Then between $\Omega$ and any generic cut $J$ outside $\Omega$, there is a $Y$-interval in which no generic cut $I^{\prime}$ satisfies $(M, I, c) \equiv\left(M, I^{\prime}, c\right)$.

Proof Without loss of generality, suppose that $(M, I, c) \equiv(M, J, c)$. It follows that $I$ and $J$ are in the same interstice over $c$, and so $\Omega$ is not an interstice over $c$. Using Corollary 9.4, take a $Y$-interval $\llbracket r, s \rrbracket$ that is disjoint from $\Omega$ but is in the same interstitial gap as $\Omega$. Let $I^{\prime}$ be a generic cut in $\llbracket r, s \rrbracket$, and let $\Omega^{\prime}$ be the constant region over $c$ containing $I^{\prime}$. Proposition 9.5 says that $\operatorname{tp}(\Omega / c) \neq \operatorname{tp}\left(\Omega^{\prime} / c\right)$. If 
$\theta(x, c) \in \operatorname{tp}(\Omega / c) \backslash \operatorname{tp}\left(\Omega^{\prime} / c\right)$, then points $x \in M$ satisfying $\theta(x, c)$ are cofinal in $I$ but not in $I^{\prime}$. Therefore $(M, I, c) \not \equiv\left(M, I^{\prime}, c\right)$.

\section{Special Cuts}

Imitating the definition of special points, we devise the notion of special cuts. It seems that an additional countability assumption on our model $M$ is needed for most of this section, because apparently we cannot avoid dealing with automorphisms.

Definition A cut $I$ is special over an element $c \in M$ if there exists an interval around $I$ in which the only cut conjugate to $I$ over $c$ is $I$ itself.

Special points and special cuts are naturally rather close to each other.

Lemma 12.1 Let $c \in M$, and let $w$ be a special point over $c$. Then either $M(w)$ or $M[w]$ is a special cut over $c$.

Proof Let $\theta(x, z) \in \mathscr{L}_{\mathrm{A}}$ that witnesses the specialness of $w$. Without loss of generality, suppose that we have $s \gg w$ satisfying

$$
M \models \theta(w, c) \wedge \forall x \in[w+1, s] \neg \theta(x, c) .
$$

We claim that no $g \in \operatorname{Aut}(M, c)$ maps $M(w)$ into $w+1, s \rrbracket$ without fixing $M(w)$. To see this, take any $g \in \operatorname{Aut}(M, c)$ making $g(M(w)) \in \llbracket w+1, s \rrbracket$. If there is $k \in \mathbb{N}$ such that $g(M(w))<Y_{k}(w) \in M(w)$, then

$$
\begin{aligned}
g\left(Y_{k}(w)\right) & =g\left(Y_{k}((\max x \in M(w))(\theta(x, c)))\right) \\
& =Y_{k}((\max x \in g(M(w)))(\theta(x, c))) \\
& =Y_{k}(w)>g(M(w)),
\end{aligned}
$$

which is not possible. Similarly, one cannot have $M(w)<g(M(w))$. Therefore $g(M(w))=M(w)$, as required.

A consequence of this lemma is a characterization of constant regions in terms of special cuts.

Proposition 12.2 Suppose that $M$ is recursively saturated. Fix a parameter $c \in M$, and let $\Omega$ be a convex subset of $M$ which satisfies

$$
\inf \Omega<M[d]<M(d)<\sup \Omega
$$

for all $d \in \Omega$. Then $\Omega$ is constant over $c$ if and only if it contains no $Y$-cut that is special over $c$.

Proof Lemmas 4.2 and 12.1 imply that if $\Omega$ is not constant over $c$, then it contains a special $Y$-cut over $c$. This proves one direction of the proposition.

Conversely, suppose that $\Omega$ is constant over $c$. Let $I$ be a $Y$-cut in $\Omega$, and let $\llbracket r, s \rrbracket$ be an interval around $I$. Without loss, assume that $\llbracket r, s \rrbracket \subseteq \Omega$. Using the axioms of an indicator, we can find a subinterval $\llbracket u, v \rrbracket \subseteq \llbracket r, s \rrbracket$ which does not contain $I$, and so by Theorem 10.1, there is $g \in \operatorname{Aut}(M, c)$ such that $g(I) \in \llbracket u, v \rrbracket$. Since the choice of $\llbracket r, s \rrbracket$ is arbitrary, the cut $I$ cannot be special over $c$.

It is conceivable that specialness is the opposite of genericity. The next theorem describes the precise relationship between these two notions. 
Theorem 12.3 Let $M$ be a countable recursively saturated model of PA. Then a $Y$-cut $I$ is generic if and only if over no element of $M$ is I a limit of special $Y$-cuts.

Proof Suppose that $I$ is a generic cut. If $c \in M$ and $\Omega$ is a constant region over $c$ containing $I$, then Proposition 12.2 says that $\Omega$ cannot contain any special $Y$-cut over $c$, and so $I$ is not a limit of such cuts.

Conversely, suppose that $I$ is not generic. Let $c \in M$ over which no constant interval can contain $I$. Then in view of Lemma 4.2, there must be special points over $c$ arbitrarily close to $I$. Note that we may assume $I$ is neither $M(d)$ nor $M[d]$ for any $d \in M$, because otherwise $I$ would itself be a special cut over $d$. So we are done by Lemma 12.1.

We also have a theorem that loosely connects specialness with genericity.

Theorem 12.4 Let $M$ be a countable recursively saturated model of PA. Then a $Y$-cut I is generic if and only if for every $c \in M$, there exists an interval containing $I$ in which the orbit of I under the action of $\operatorname{Aut}(M, c)$ is $Y$-dense.

Proof The "only if" part is an easy consequence of Theorem 10.1. The argument we use for the "if" part is similar to that of [6, Theorem 3.10]. Let $c \in M$. Pick an interval $\llbracket r, s \rrbracket$ containing $I$ in which the orbit of $I$ under the action of $\operatorname{Aut}(M, c)$ is dense. Using Theorem 3.1, find a subinterval $\llbracket a, b \rrbracket \subseteq \llbracket r, s \rrbracket$ that is constant over $c$. By hypothesis, there is $g \in \operatorname{Aut}(M, c)$ mapping $I$ into $\llbracket a, b \rrbracket$, and so it makes $g^{-1}(\llbracket a, b \rrbracket)$ a constant interval over $c$ containing $I$.

\section{Existentially Closed Cuts}

Let us briefly recapitulate Kaye and Wong [7]. We work with the language $\mathscr{L}_{\text {cut }}$, which, in addition to the symbols in $\mathscr{L}_{\mathrm{A}}$, contains a new function symbol for each $\mathscr{L}_{\mathrm{A}}$-Skolem function, and a new unary predicate symbol $\mathbb{I}$ intended to interpret a cut. Recall from Section 2 that each $Y_{k}$, where $k \in \mathbb{N}$, is assumed to be parameter-free definable. So they make sense across a class $\mathbf{K}$ of models of PA containing $M$. This class $\mathbf{K}$ will be additionally required to be cofinal in the class of all models of PA under elementary embeddings. In other words, we want every model of PA to have an elementary extension in $\mathbf{K}$. The theory $\mathrm{PA}_{Y}$ consists of the axioms of PA, the definitions of the $\mathscr{L}_{\mathrm{A}}$-Skolem functions, an axiom saying that $\mathbb{I}$ is a proper cut, and a scheme saying that $\mathbb{I}$ is closed under $Y_{k}$ for all $k \in \mathbb{N}$.

Given a cut $I$, the existential type of an element $c \in M$, denoted by $\operatorname{etp}_{(M, I)}(c)$, is the set of all $\exists_{1}$-formulas satisfied by $c$ in $(M, I)$. Kaye and Wong observed in [7] that over $\mathrm{PA}_{Y}$, every $\exists_{1}$-formula $\theta(z) \in \mathscr{L}_{\text {cut }}$ says that

$$
\mathbb{I} \text { is not closed under the function } x \mapsto F(x, z)
$$

for some $\mathscr{L}_{\mathrm{A}}$-Skolem function $F$. So, for example, if $M$ is recursively saturated and $\Omega$ is a constant region over $c$, then Corollary 7.4 says that $\operatorname{etp}_{(M, \inf \Omega)}(c)=$ $\operatorname{etp}_{(M \text {,sup } \Omega)}(c)$. An existentially closed model of $\mathrm{PA}_{Y}$ is a model $(M, I) \models \mathrm{PA}_{Y}$ such that whenever $(K, J)$ is an extension of $(M, I)$ satisfying $\mathrm{PA}_{Y}$, where $K \in \mathbf{K}$, we have

$$
(K, J) \models \theta(c) \quad \Rightarrow \quad(M, I) \models \theta(c)
$$


for all $\exists_{1}$-formulas $\theta(z) \in \mathscr{L}_{\text {cut }}$ and all $c \in M$. Abusing the notation slightly, I will sometimes write "the $Y$-cut $I$ is existentially closed" when I mean " $(M, I)$ is an existentially closed model of PA $Y$." General model-theoretic facts tell us existentially closed models of $\mathrm{PA}_{Y}$ exist, because $\mathrm{PA}_{Y}$ is $\forall_{2}$.

Thanks to the following proposition, we can work on existentially closed models of $\mathrm{PA}_{Y}$ without mentioning extensions and the class $\mathbf{K}$ in the later sections.

Proposition 13.1 Let I be a proper $Y$-cut. Then $(M, I)$ is an existentially closed model of $\mathrm{PA}_{Y}$ if and only if for every $\mathscr{L}_{\mathrm{A}}$-definable function $F$ under which $I$ is closed, there is $k \in \mathbb{N}$ such that $Y_{k}$ dominates $F$ on $I$.

Proof Let $F$ be a definable function on $M$ under which $I$ is closed. Without loss of generality, suppose that $F$ is increasing. Assume that $F$ is not dominated by $Y_{k}$ on $I$ for any $k \in \mathbb{N}$. Using this assumption and the compactness theorem, find an elementary extension $K \succ M$ that contains two new elements $r, s$ satisfying

$$
x<r \ll s \leqslant F(r)<y
$$

for all $x \in I$ and all $y \in M \backslash I$. By passing to an elementary extension if necessary, we may suppose that $K \in \mathbf{K}$. Let $J$ be any $Y$-cut in $K$ between $r$ and $s$. Then $(K, J) \supseteq(M, I)$, but $J$ is not closed under $F$. So $(M, I)$ is not existentially closed.

Conversely, fix some $(K, J) \supseteq(M, I)$, where $K \in \mathbf{K}$ and $(K, J) \models \mathrm{PA}_{Y}$. Let $F$ be a definable function on $M$ under which $I$ is closed. Without loss, we assume that $F$ is increasing again. Using the hypothesis, find $k \in \mathbb{N}$ such that $Y_{k}$ dominates $F$ on $I$. Then there is $\llbracket a, b \rrbracket \subseteq M$ containing $I$ which makes

$$
M \models \forall x \in \llbracket a, b \rrbracket Y_{k}(x) \geqslant F(x) .
$$

This transfers to $K$ by elementarity. So $Y_{k}$ dominates $F$ on $J$ too. In particular, $J$ is closed under $F$ because $J$ is a $Y$-cut.

There are numerous corollaries to this proposition, the most straightforward one being the following. Recall from Kirby [8] that the $Y$-index of a cut $I$ is defined by

$$
\operatorname{index}_{Y}(I)=\{n \in M: \forall x \in I \forall y>I Y(x, y) \geqslant n\} .
$$

The $Y$-index of a $Y$-cut is always bigger than $\mathbb{N}$.

Corollary 13.2 Suppose that we have an existentially closed model $(M, I) \models$ $\mathrm{PA}_{Y}$. Then $\operatorname{index}_{Y}(I)=\mathbb{N}$. In particular, $(M, I)$ is not $\forall_{1}$-recursively saturated.

The following corollary was first proved in [7].

Corollary 13.3 If $M$ is recursively saturated and I is a generic cut, then $(M, I)$ is an existentially closed model of $\mathrm{PA}_{Y}$.

Proof Combine Theorem 8.1 and Proposition 13.1.

Taking away the arithmetic saturation part from Kaye's proof of Theorem 3.1, we obtain the next proposition.

Proposition 13.4 Let $M$ be a countable nonstandard model of PA. Then for every $\llbracket a, b \rrbracket \subseteq M$, there is a cut I of $M$ contained in $\llbracket a, b \rrbracket$ such that $(M, I)$ is an existentially closed model of $\mathrm{PA}_{Y}$. 
Proof Enumerate all parametrically definable functions $M \rightarrow M$ in the sequence $\left(F_{n}\right)_{n \in \mathbb{N}}$. We will construct, recursively, an $\omega$-sequence of intervals

$$
\llbracket a, b \rrbracket=\llbracket a_{0}, b_{0} \rrbracket \supseteq \llbracket a_{1}, b_{2} \rrbracket \supseteq \llbracket a_{2}, b_{2} \rrbracket \supseteq \cdots
$$

such that $\left(M, \sup \left\{a_{n}: n \in \mathbb{N}\right\}\right)$ is an existentially closed model of PAY. Suppose that we already have $\llbracket a_{n}, b_{n} \rrbracket$. Consider $F_{n}$. There are two possibilities.

(i) There is $\llbracket r, s \rrbracket \subseteq \llbracket a_{n}, b_{n} \rrbracket$ such that $F_{n}(r)=s$.

(ii) For every $r \in \llbracket a_{n}, b_{n} \rrbracket$, we have $Y\left(r, F_{n}(r)\right) \in \mathbb{N}$.

In case (i), no $Y$-cut in $\llbracket r, s \rrbracket$ can be closed under $F_{n}$. So we can safely let $\llbracket a_{n+1}, b_{n+1} \rrbracket$ be any such $\llbracket r, s \rrbracket$. In case (ii), there must be $k \in \mathbb{N}$ such that

$$
M \models \forall r \in \llbracket a_{n}, b_{n} \rrbracket Y\left(r, F_{n}(r)\right) \leqslant k .
$$

Thus, any $Y$-cut $I$ in $\llbracket a_{n}, b_{n} \rrbracket$ is closed under $F_{n}$. Furthermore, if $(M, I) \subseteq(K, J) \models$ PA $_{Y}$ where $I \in \llbracket a_{n}, b_{n} \rrbracket$ and $K \in \mathbf{K}$, then $J$ must be closed under $F_{n}$ too, because $(*)$ transfers to $K$. So we can set $\llbracket a_{n+1}, b_{n+1} \rrbracket$ to $\llbracket a_{n}, b_{n} \rrbracket$. In either case, the function $F_{n}$ cannot be a counterexample to the existential closedness of the outcome. Since every definable function is considered in this construction, we conclude that $\left(M, \sup \left\{a_{n}: n \in \mathbb{N}\right\}\right)$ is existentially closed.

Apparently, it is not impossible that for every cut $I$, one can find a suitable indicator $Y$ to make $(M, I)$ an existentially closed model of $\mathrm{PA}_{Y}$. In view of Proposition 13.1, this is related to the following question.

Question 13.5 Is there a cut $I$ for which no cofinal subclass of $\mathfrak{F}_{I}$ is coded in $M$ ? Here $\mathfrak{F}_{I}$ denotes the class of all parameter-free definable functions $M \rightarrow M$ under which $I$ is closed, ordered under domination on $I$.

\section{$14 \omega$-Limits}

Probably the simplest cuts are those of the form

$$
\sup \left\{(a)_{n}: n \in \mathbb{N}\right\} \quad \text { or } \quad \inf \left\{(b)_{n}: n \in \mathbb{N}\right\},
$$

where $a, b \in M$. None of these cuts turns out to be existentially closed. We start with a slightly more general proposition which goes in the direction of Question 13.5.

Proposition 14.1 For $a$ Y -cut I and a monotonically increasing definable function $f: M \rightarrow M$, the following are equivalent:

(a) $I$ is closed under $x \mapsto Y_{f(x)}(x)$;

(b) $f(I) \subseteq \operatorname{index}_{Y}(I)$.

Proof If $f(I) \subseteq \operatorname{index}_{Y}(I)$, then clearly $I$ is closed under $x \mapsto Y_{f(x)}(x)$. Conversely, suppose that $I$ is closed under $x \mapsto Y_{f(x)}(x)$. Let $a \in I$. Then for each $x \in I$ above $a$ and each $y>I$,

$$
\begin{aligned}
Y(x, y) & \geqslant Y\left(x, Y_{f(x)}(x)\right) \quad \text { since } Y_{f(x)}(x) \in I<y, \\
& \geqslant Y\left(x, Y_{f(a)}(x)\right) \quad \text { since } a<x \text { and } f, Y \text { are monotone, } \\
& =f(a) .
\end{aligned}
$$

Therefore $f(a) \in \operatorname{index}_{Y}(I)$. 
Lemma 14.2 If I is a $Y$-cut of the form

$$
\sup \left\{(a)_{n}: n \in \mathbb{N}\right\}
$$

where $a \in M$, then $(M, I)$ is not an existentially closed model of $\mathrm{PA}_{Y}$.

Proof Without loss of generality, suppose that $(a)_{n}<(a)_{n+1}$ for all $n \in \mathbb{N}$. We may also assume that $\operatorname{index}_{Y}(I)=\mathbb{N}$ because of Corollary 13.2. If $f$ is a monotonically increasing definable function $M \rightarrow M$ such that $f(I)=\mathbb{N}$, then $x \mapsto Y_{f(x)}(x)$ dominates $Y_{k}$ on $I$ for every $k \in \mathbb{N}$. Therefore, by Propositions 13.1 and 14.1, it suffices to find a monotonically increasing definable function $f: M \rightarrow M$ such that $f(I)=\mathbb{N}$. One example is given by $f(x)=(\mu n)\left((a)_{n} \geqslant x\right)$.

Lemma 14.3 If I is a $Y$-cut of the form

$$
\inf \left\{(b)_{n}: n \in \mathbb{N}\right\}
$$

where $b \in M$, then $(M, I)$ is not an existentially closed model of $\mathrm{PA}_{Y}$.

Proposition 14.1 does not help in proving this lemma, because there is no definable increasing function $f$ mapping a downward $\omega$-limit to $\mathbb{N}$. Moreover, we do not have an analogue of Proposition 14.1 for a monotonically decreasing function $f$ satisfying $f(I) \subseteq \subseteq_{\mathrm{dcf}} M \backslash \operatorname{index}_{Y}(I)$. To see this, consider, for example, $I=M[b]$ for some $b \in M$, and $f(x)=(\mu k)\left(Y_{k}(x) \geqslant b\right)$. Since $f(M \backslash I) \subseteq \mathrm{cf} \mathbb{N}$, we have $f(I) \subseteq$ dcf $M \backslash \mathbb{N}$ by underspill. However, for all $x \in I$,

$$
Y_{f(x)}(x)=Y_{(\mu k)\left(Y_{k}(x) \geqslant b\right)}(x) \geqslant b>I .
$$

Fortunately, the trick used in Kaye [4, Proposition 2.4] works.

Proof Without loss of generality, assume that $(b)_{n+1}<(b)_{n}$ for all $n \in \mathbb{N}$. We may also suppose that $Y\left((b)_{n+1},(b)_{n}\right) \in \mathbb{N}$ for all $n \in \mathbb{N}$. This can be seen using Proposition 13.1 with the function

$$
x \mapsto(\mu y)\left(\exists n\left(y=(b)_{n} \wedge(b)_{n+1} \geqslant x\right)\right)
$$

or be shown directly from the definitions. Define

$$
F(x)=(\mu z)\left(Y(x, z) \geqslant Y\left(z,(b)_{0}\right)\right) .
$$

One can verify using condition (3) on page 604 that whenever $x \in I$, both $[x, F(x)]$ and $\left[F(x),(b)_{0}\right]$ are $Y$-large. Hence $I$ is closed under $F$ but $F$ dominates $Y_{k}$ on $I$ for every $k \in \mathbb{N}$. Proposition 13.1 then says that $(M, I)$ is not an existentially closed model of $\mathrm{PA}_{Y}$.

In Corollary 8.2, we saw that the infima of constant regions share some common properties with existentially closed $Y$-cuts. However, Corollary 6.2 and Lemma 14.2 tell us that none of these cuts are existentially closed. In view of Lemma 14.3, the same is true for the suprema of constant regions. 


\section{Genericity and Existential Closedness}

We conclude this paper with a few new characterizations of generic cuts in terms of existential closedness. The following lemma will be handy.

Lemma 15.1 Suppose that $M$ is recursively saturated. Fix an element $c \in M$ and an existentially closed model $(M, I) \models \mathrm{PA}_{Y}$. Let $\llbracket r, s \rrbracket$ be an interval around $I$ which avoids all points of the form

$$
(\max x \in I)(\theta(x, c)) \quad \text { or } \quad(\min x>I)(\theta(x, c)) \text {, }
$$

where $\theta(x, z)$ is some $\mathscr{L}_{\mathrm{A}}$-formula. Then $\llbracket r, s \rrbracket$ is constant over $c$.

Proof We claim that if $\varphi(x, c)$ is an $\mathscr{L}_{\mathrm{A}}$-formula satisfied by some element of $\llbracket r, s \rrbracket$, then it has to be satisfied in every $Y$-large subinterval of $\llbracket r, s \rrbracket$. By recursive saturation, this is sufficient to finish the proof. There are three possibilities:

(i) $(M, I) \models \forall x \in \mathbb{I} \neg \varphi(x, c)$;

(ii) $(M, I) \models \exists w(w=(\max x \in \mathbb{I})(\varphi(x, c)))$;

(iii) $(M, I) \models Q x \in \mathbb{I} \varphi(x, c)$.

In case (i), we must have $M \models \forall x \in \llbracket r, s \rrbracket \neg \varphi(x, c)$ because of our choice of $\llbracket r, s \rrbracket$. For a similar reason, if (ii) holds, then $(\max x \in \mathbb{I})(\varphi(x, c))<r$ and so no $x \in \llbracket r, s \rrbracket$ can satisfy $\varphi(x, c)$. Consider case (iii). By Proposition 13.1, there is $k \in \mathbb{N}$ such that $Y_{k}$ dominates the function

$$
x \mapsto\left(\mu x^{\prime}>x\right)\left(\varphi\left(x^{\prime}, c\right)\right)
$$

on $I$. By our hypothesis on $\llbracket r, s \rrbracket$, this domination must start below $r$. Therefore, we have $M \models \forall x \in \llbracket r, s \rrbracket \exists x^{\prime} \in\left[x, Y_{k}(x)\right] \varphi\left(x^{\prime}, c\right)$ for some $k \in \mathbb{N}$, as required.

Theorem 15.2 Let $(M, I)$ be an existentially closed model of $\mathrm{PA}_{Y}$ in which $M$ is recursively saturated. The following are equivalent.

(a) I is a generic cut.

(b) $(M, I)$ is $\exists_{1}$-recursively saturated and $\operatorname{etp}_{(M, I)}(c)$ is coded for all $c \in M$.

(c) There is a lower bound for

$$
\left\{(\min x>I)(\theta(x, c)): \theta \in \mathscr{L}_{\mathrm{A}} \text { for which the min exists }\right\}
$$

above I for every $c \in M$.

(d) There is an upper bound for

$$
\left\{(\max x \in I)(\theta(x, c)): \theta \in \mathscr{L}_{\mathrm{A}} \text { for which the max exists }\right\}
$$

in I for every $c \in M$.

(e) For every $c \in M$, there exists $b>I$ such that for all functions $F: M \rightarrow M$ definable over $c$, we have

$$
\forall x \in I F(x) \leqslant b \quad \Rightarrow \quad \forall x \in I F(x) \in I .
$$

(f) For every $c \in M$, there exists $a \in I$ such that for all functions $F: M \rightarrow M$ definable over $c$, we have

$$
\forall x<a F(x) \in I \quad \Rightarrow \quad \forall x \in I F(x) \in I .
$$

Clauses (b)-(f) are true whenever $(M, I)$ is an $\forall_{1}$-recursively saturated model

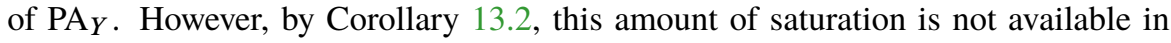
existentially closed cuts. 
Proof Corollary 7.2 says that (c) $\Leftrightarrow$ (e) and (d) $\Leftrightarrow$ (f). So we can ignore (e) and (f).

We first show that (c) $\Rightarrow$ (d). Pick any $c \in M$. Using (c), take $s>I$ that is above every $(\min x>I)(\theta(x, c))$ where $\theta \in \mathscr{L}_{\mathrm{A}}$ for which the minimum exists. A further application of (c) to the parameter $s$ tells us that $I \neq M[s]$. Let $r^{\prime}>I$ which satisfies $r^{\prime} \ll s$. We claim that $(\max x \in I)(\theta(x, c))$ exists if and only if

$$
M \models \forall x \in \llbracket r^{\prime}, s \rrbracket \neg \theta(x, c) \wedge \exists x<s \theta(x, c)
$$

for every $\mathscr{L}_{\mathrm{A}}$-formula $\theta(x, z)$. For the "only if" part, suppose that $(\max x \in I)(\theta(x$, $c)$ ) exists. Then clearly $M \models \exists x<s \theta(x, c)$. By overspill, we also know that

$$
(M, I) \models \exists b>\mathbb{I} \forall x>\mathbb{I}(x<b \rightarrow \neg \theta(x, c)) .
$$

If $(M, I) \models \forall x>\mathbb{I} \neg \theta(x, c)$, then we are done. So it remains to consider the case when $(\min x>I)(\theta(x, c))$ exists. If this minimum exists, then it is bigger than $s$ by the choice of $s$, and so there cannot be any $x \in \llbracket r^{\prime}, s \rrbracket$ that satisfies $\theta(x, c)$. This is what we want. For the "if" part, suppose that $M \models \forall x \in \llbracket r^{\prime}, s \rrbracket \neg \theta(x, c) \wedge \exists x<s \theta(x, c)$. Note that since $s$ is smaller than all the minima, there must be some $x \in I$ which satisfies $\theta(x, c)$. We apply the same trick as in the previous proof: define

$$
F(x)=\left(\mu x^{\prime}>x\right)\left(\theta\left(x^{\prime}, c\right)\right) .
$$

If $I$ is not closed under $F$, then we are done. So suppose not. Using Proposition 13.1, find $k \in \mathbb{N}$ such that $Y_{k}$ dominates $F$ on $I$. By our choice of $s$, we must have $F(x) \leqslant Y_{k}(x)$ for all $x>I$ below $s$. In particular, $M \models \exists x \in \llbracket r^{\prime}, s \rrbracket \theta(x, c)$. This is contradictory to our hypothesis. Thus $I$ cannot be closed under $F$ after all. This finishes the proof of our claim. It thus follows from recursive saturation that there is $a \in M$ making

$$
\left\{(a)_{n}: n \in \mathbb{N}\right\}=\left\{(\max x \in I)(\theta(x, c)): \theta \in \mathscr{L}_{\mathrm{A}} \text { for which the max exists }\right\} .
$$

Lemma 14.2 says that these maxima cannot be cofinal in $I$, showing (d).

Similarly, one shows that (d) $\Rightarrow$ (c). Therefore (c) $\Rightarrow$ (a) and (d) $\Rightarrow$ (a) by Lemma 15.1. We will prove that (a) $\Rightarrow$ (b) and (b) $\Rightarrow$ (c).

The fact that (a) $\Rightarrow$ (b) was first proved in [7]. We include an alternative proof here using the machinery developed in this paper. Suppose that $I$ is a generic cut. The codedness part is already taken care of by Lemma 5.1. For the recursive saturation part, consider the recursive $\exists_{1}$-type

$$
p(w)=\left\{\exists \llbracket x, y \rrbracket \ni \mathbb{I} \varphi_{i}(w, x, y, c): i \in \mathbb{N}\right\},
$$

where $c$ is some element of $M$. Take any constant interval $\llbracket a, b \rrbracket$ over $c$ around $I$. Since $p$ is finitely satisfied in $(M, I)$, we have

$$
M \models \exists[u, v] \subseteq \llbracket a, b \rrbracket\left(Y(u, v)>k \wedge \exists w \exists[x, y] \supseteq[u, v] / \varphi_{i<k}(w, x, y, c)\right),
$$

for every $k \in \mathbb{N}$. Using overspill, one can find $\llbracket u, v \rrbracket \subseteq \llbracket a, b \rrbracket \stackrel{i<k}{\text { such }}$ that

$$
M \models \exists w \exists[x, y] \supseteq \llbracket u, v \rrbracket \bigwedge_{i \in \mathbb{N}} \varphi_{i}(w, x, y, c) .
$$

So $p(w)$ is realized in $(M, I)$ by Theorem 10.1 . 
Finally, consider the implication (b) $\Rightarrow$ (c). Fix $c \in M$. It suffices to realize

$$
\begin{aligned}
p(u, v)= & \left\{\exists x \in \mathbb{I} \exists y>\mathbb{I} \varphi(x, y, c) \rightarrow \exists x<u \exists y>v \varphi(x, y, c): \varphi \in \mathscr{L}_{\mathrm{A}}\right\} \\
& \cup\{u \in \mathbb{I}<v\} .
\end{aligned}
$$

Since $\operatorname{etp}_{(M, I)}(c)$ is coded, this $p(u, v)$ is equivalent to a recursive $\exists_{1}$-type. So we are done by $\exists_{1}$-recursive saturation.

Recall from Corollary 8.4 that the existence of generic cuts implies the strength of $\mathbb{N}$. Thus if $M$ is a countable model of PA in which $\mathbb{N}$ is not strong, and if $I$ is an existentially closed $Y$-cut given by Proposition 13.4, then all of (a)-(f) in Theorem 15.2 must fail for $I$.

\section{Note Added in Proof}

The argument on page 154 of J. Hirschfeld and W. H. Wheeler's Forcing, Arithmetic, Division Rings (vol. 454 of Lecture Notes in Mathematics, Springer, Berlin, 1975) shows that the saturation condition in Theorem 15.2(b) is redundant.

\section{References}

[1] Bamber, N., and H. Kotlarski, "On interstices of countable arithmetically saturated models of Peano arithmetic," Mathematical Logic Quarterly, vol. 43 (1997), pp. 525-40. Zbl 0884.03042. MR 1477620. DOI 10.1002/malq.19970430408. 605, 608, 612, 624

[2] Bigorajska, T., H. Kotlarski, and J. H. Schmerl, "On regular interstices and selective types in countable arithmetically saturated models of Peano Arithmetic," Fundamenta Mathematicae, vol. 158 (1998), pp. 125-46. Zbl 0920.03069. MR 1656934. 605, 610, 612

[3] Kaye, R., Models of Peano Arithmetic, vol. 15 of Oxford Logic Guides, Oxford University Press, New York, 1991. MR 1098499. 604

[4] Kaye, R., "Generic cuts in models of arithmetic," Mathematical Logic Quarterly, vol. 54 (2008), pp. 129-44. Zbl 1138.03031. MR 2402622. DOI 10.1002/malq.200710017. 603, 604, 605, 615, 620

[5] Kaye, R., R. Kossak, and H. Kotlarski, "Automorphisms of recursively saturated models of arithmetic," Annals of Pure and Applied Logic, vol. 55 (1991), pp. 67-99. Zbl 0748.03023. MR 1134917. DOI 10.1016/0168-0072(91)90098-7. 612

[6] Kaye, R., and T. L. Wong, "Truth in generic cuts," Annals of Pure and Applied Logic, vol. 161 (2010), pp. 987-1005. Zbl 1230.03070. MR 2629502. DOI 10.1016/j.apal.2009.11.001. 603, 604, 607, 611, 615, 617

[7] Kaye, R., and T. L. Wong, "The model theory of generic cuts," to appear in Logic Without Borders: Essays on Set Theory, Model Theory, Philosophical Logic, and Philosophy of Mathematics, edited by J. Kennedy, R. Kossak, T. Hyttinen, A. Villaveces, and M. Kesälä, De Gruyter, Berlin. Zbl 06305053. DOI 10.1515/9781614516873. 604, 617, 618,622

[8] Kirby, L. A. S., "Initial segments of models of arithmetic," Ph.D. dissertation, University of Manchester, Manchester, UK, July 1977. 618

[9] Kossak, R., and J. H. Schmerl, The Structure of Models of Peano Arithmetic, vol. 50 of Oxford Logic Guides, Oxford University Press, Oxford, 2006. Zbl 1101.03029. MR 2250469. DOI 10.1093/acprof:oso/9780198568278.001.0001. 604, 614

[10] Schmerl, J. H., "Moving intersticial gaps," Mathematical Logic Quarterly, vol. 48 (2002), pp. 283-96. Zbl 0997.03036. MR 1883243. 
DOI 10.1002/1521-3870(200202)48:2<283::AID-MALQ283>3.3.CO;2-R. $\quad 605$, 612

[11] Wong, T. L., "Generic cuts in a general setting," Master's thesis (qualifying), University of Birmingham, Birmingham, UK, 2008. 614

\section{Acknowledgments}

I would like to thank Richard Kaye for many stimulating discussions on and around this topic. I am particularly grateful to him for pointing me to the work of Bamber and Kotlarski [1]. The results in Section 4 were found when I was studying for my Ph.D. at the University of Birmingham under Kaye's supervision.

I would also like to thank the organizers of the Model Theory and Proof Theory of Arithmetic conference, especially Roman Kossak, for inviting me. This conference, which was held in Będlewo, Poland in July 2012, was where I gathered enough momentum to start writing this paper.

A large part of the research reported here was carried out when I was financially supported by the John Templeton Foundation under the Philosophical Frontiers in Reverse Mathematics project.

Department of Mathematics

Ghent University

Ghent

Belgium

wtl@cage.ugent.be 